\title{
Implementation of Communication Interfaces in Power Converters
}

\author{
Snehal R. Watharkar ${ }^{\# 1}$, Mahadev S. Patil ${ }^{\# 2}$ \\ \# Department of Electronics and Telecommunication Engineering, Shivaji University, Kolhapur \\ Rajarambapu Institute of Technology, Islampur, Sangli, Maharashtra, India \\ ${ }^{1}$ snehal.watharkar@ritindia.edu \\ 2mahadev.patil@ritindia.edu
}

\begin{abstract}
This paper presents various communication interfaces used to display electrical parameters and warning messages of power converters using RL78/G14 microcontroller of Renesas make. 128x64 Graphic LCD is used to display real time electrical parameters of single phase inverter. 320x240 GLCD is used to display and control electrical parameters of Three Phase Active filter and Static Voltage Regulator. GSM modem is used to send the fault and warning messages. USB protocol is used to download electrical parameters from power converters. Simple Network Management Protocol (SNMP) card is used to monitor parameters through web access. Single phase inverters are used for domestic as well as commercial purposes whereas Three Phase Active Filter \& Static Voltage Regulator are used in industry.
\end{abstract}

Keyword - RL78/G14, GLCD module, GSM, USB, SNMP

\section{INTRODUCTION}

In serial communication, single bit of data is transmitted at a time, sequentially, to the receiver. It is mainly used for low data transfer rate and long distance communication. UART is the serial communication protocol used for asynchronous data communication between computer and peripheral devices [1]. In parallel communication, numbers of bits are transmitted at a time, to the receiver. It is faster than serial communication but it is not used for long distance communication. In this paper, serial communication with power converters is achieved by GSM/USB/SNMP and parallel communication is achieved by graphical LCD. It describes various communication standards used for Modern Power Electronics Equipment like Inverter, UPS, Active Filter, Static Voltage Regulator etc. to monitor real time process parameters \& error messages through USB / Ethernet / GSM / SNMP. These power converters are used for domestic and commercial applications.

Single Phase Inverter System is used in domestic applications to supply power to critical loads under utility power failure. Three Phase Static Voltage Regulators are used to regulate the voltage applied to critical loads under utility voltage disturbances like surges and sags. Three Phase Active Filter is used in industrial applications compensate for reactive power and harmonic currents drawn to the industrial load.

In this paper, USB is used to download data from the equipment to PC for analysis of causes of faults. It is fast and flexible interface which can be used to connect electrical devices to a PC and it has become one of the most popular device interfaces [2] [9]. GSM is used to send the error or status information message to the mobile station [3]. SNMP protocol is used to set the parameters and monitor the status [4] [5]. The Graphical LCD panel is used to display status summary of the equipment. A set of push buttons are provided to change the screen to input, output etc. Once fault occurs, the screen will automatically show the fault in blinking form.

Renesas RL78/G14 microcontroller is interfaced with Graphical LCD, GSM and USB for communication [8]. Software is written in Embedded C and Cube Suite is used as IDE [7].

\section{Methodology}

The microcontroller of the Power Electronics equipment contains the software for control and monitoring of various parameters. The data residing in various locations of the microcontroller memory are accessed and converted to serial form and then converted using the Interface Devices represented by ID. The software for these functions also resides in the same microcontroller. UART channel (Transmit and Receive) is used for serial communication and 8 GPIO pins are required to transfer the data using parallel communication. Fig. 1. shows the system block diagram. 


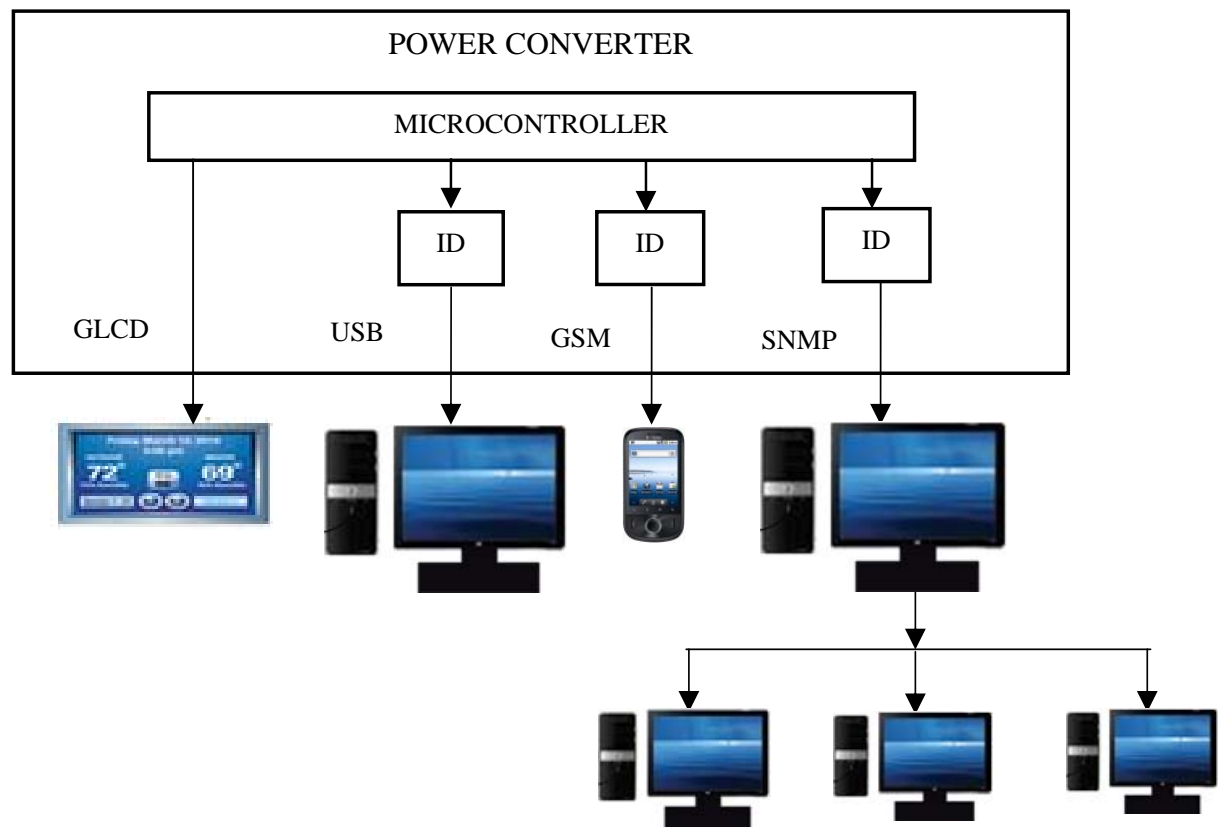

Fig. 1. System Block Diagram

\section{HARDWARE IMPLEMENTATION}

The system design has following sub-modules:

\section{A. Graphical LCD 128x64}

Oriole Graphic LCD Module 128x64 is used to display real time parameters of single phase inverter. There are 128 x 64 dots (pixels). Vertical 64 bits are divided into pages of 8 bits. So there are 8 pages addressed by X address. Horizontal 128 pixels are divided into group of two as chip1 and chip2 of 64 pixels each and addressed by Y address. After port initialization and GLCD initialization, Page X address and Y address of particular chip is selected to write single pixel.

When inverter is operated on mains supply, mains voltage, battery voltage \& battery current (charging) parameters are displayed on GLCD. When it is operated on battery supply then battery voltage, battery current (discharging), output voltage, output current $\&$ input voltage are displayed. All these electrical parameters are displayed after every 2sec. If any protection mode is activated then protection message is immediately displayed on GLCD. Analog input parameters of Power converters are sensed by appropriate transducers. Voltages are sensed by potential transformers \& currents are sensed by shunts. The outputs of transducers are converted to digital form using ADC and stored to the memory location of microcontroller. This digital count is multiplied by scaling factor and then transferred to data port of GLCD. Fig. 2. shows interfacing of GLCD with microcontroller to display electrical parameters of inverter.

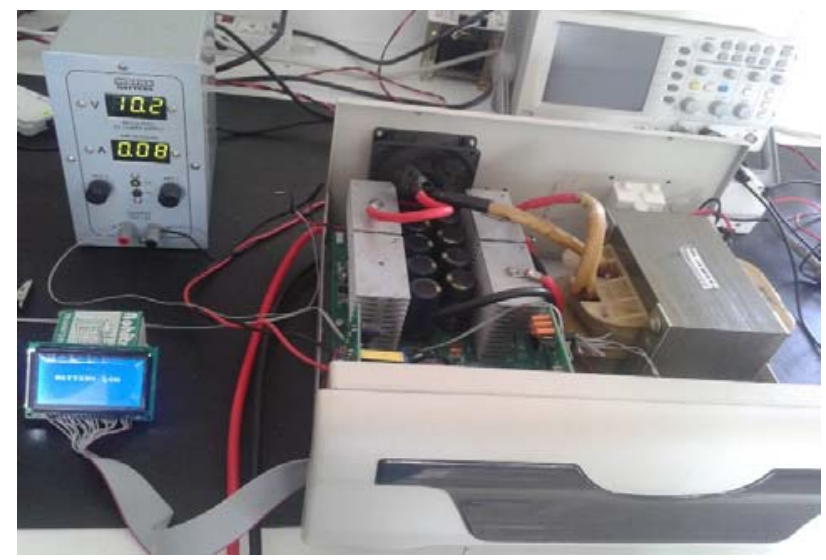

Fig. 2. OGM128x24 interfacing 


\section{B. Graphical LCD $320 \times 240$}

JHD 320x240 GLCD module is interfaced with microcontroller RL78/G14 to display real time and controlled electrical parameters. It is used to display electrical parameters of static voltage regulator \& three phase active filter. Input Parameters like input voltage input current \& input frequency, output voltage, DC voltage \& warning messages are displayed on GLCD. Under fault conditions, the display blinks continuously. Some electrical parameters like system voltage, input voltage range \& output voltage range are controlled by push buttons \& displayed. Fig. 3. shows interfacing of GLCD with microcontroller.

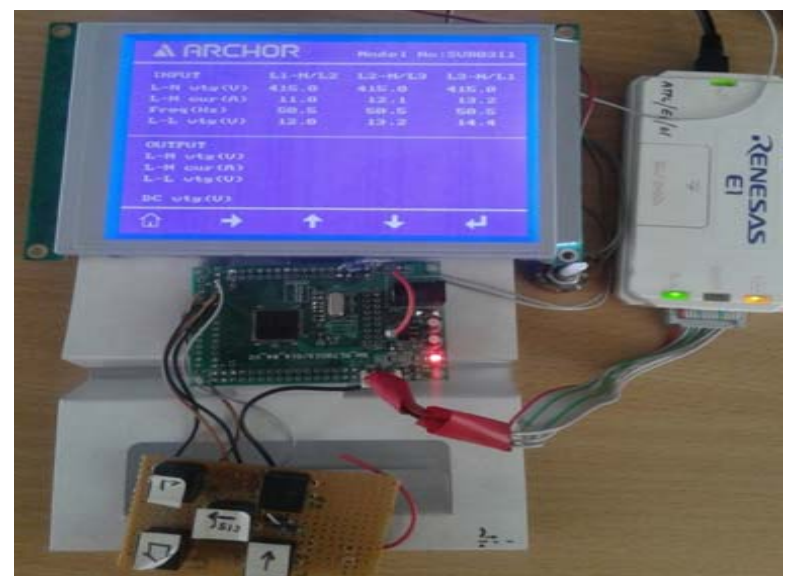

Fig. 3. JHD320x240 interfacing

\section{C. $G S M$}

SIM900 GSM modem is used to send message whenever fault occurs. It is directly interfaced with microcontroller as it is designed with 5V DC TTL interfacing circuitry. Using standard set of AT commands it is configured for baud rate from 9.6Kbps. The modem is interfaced with a microcontroller using UART (Serial communication) channel. Microcontroller is interfaced with GSM modem using UART receive and transmit pins via MAX232 level converter. It is used to convert RS232 voltage levels to TTL voltage levels and vice versa. Fig. 4. shows interfacing of GSM module with microcontroller.

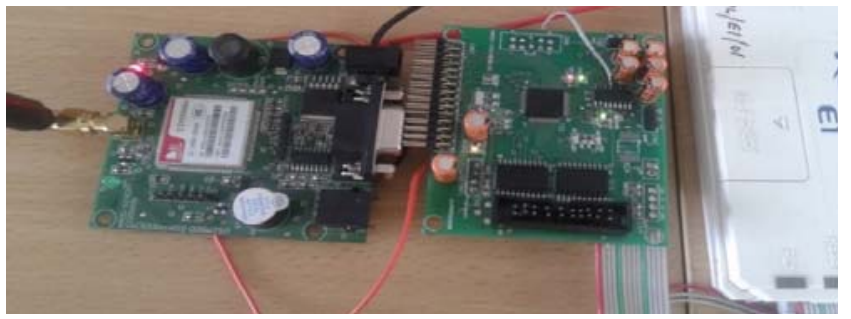

Fig. 4. GSM Interfacing

\section{USB}

USB is used to download data from power electronic converters to PC. Microcontroller has UART channel for communication. So serial UART to USB converter is used to convert UART signal into signal corresponds to USB. FT230XS converter is used for UART to USB signal conversion. Receive pin of the microcontroller is connected to transmit pin of the FT230XS converter. Transmit pin of microcontroller is connected to receive pin of FT230XS converter. Fig. 5. shows interfacing of FT230XS converter with microcontroller.

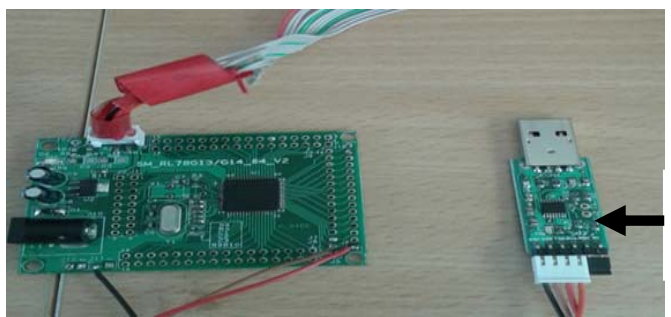

FT230XS

Converter

Fig. 5. USB interfacing 


\section{E. SNMP}

SNMP card is plug and play hardware with SNMPv1 agent and web server for UPS. Card has built-in Management Information Base (MIB).UPS can be directly accessed through any web browser from the network, since the card has a built in web server that can display UPS parameters using html files stored inside the card. Web interface is also used to configure the card and Network Management System (NMS) interface. Any status change in the UPS that corresponds to one of the SNMP traps supported by the agent, sent to all of the configured PCs immediately. IpPowerSE software is executed on Manager PC to monitor and control UPS. It can monitor several UPS in the same network at same time. It is used to display status of UPS and its parameters with graphics [6].

SNMP card is interfaced with PC using USB cable and LAN cable. It is connected to UPS system using RS232 interface. Fig. 6. shows interfacing of SNMP card with PC \& Inverter.

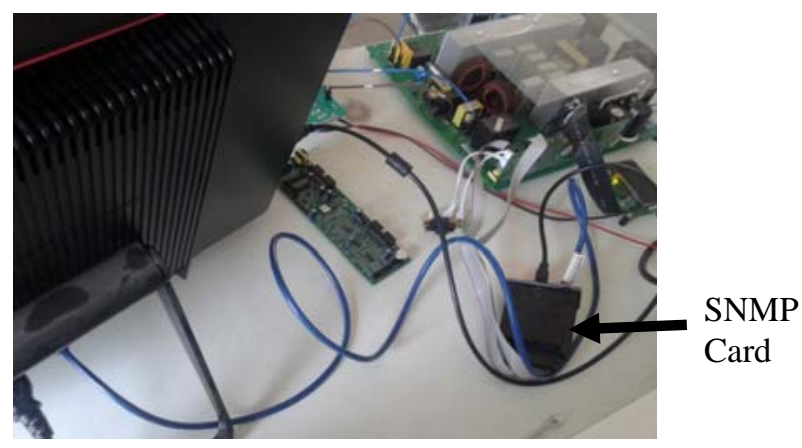

Fig. 6. SNMP card interfacing

\section{SOFTWARE IMPLEMENTATION}

\section{A. GLCD $128 \times 64$}

When inverter is operated on mains supply, mains voltage, battery voltage \& battery current (charging) parameters are displayed on GLCD. When it is operated on battery supply then battery voltage, battery current (discharging), output voltage, output current $\&$ input voltage are displayed. Fig. 7. shows the programming of 128x64 GLCD.

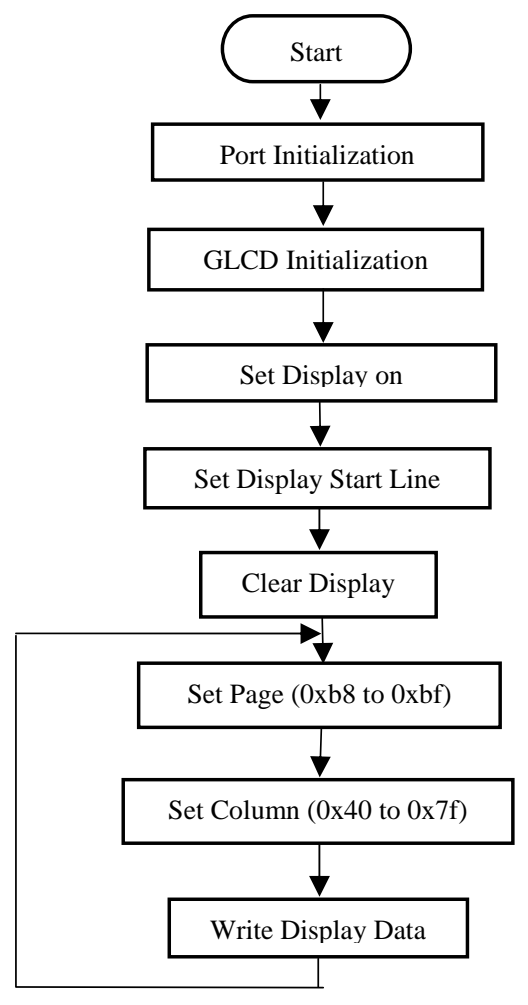

Fig. 7. Programming of $128 x 64$ GLCD 


\section{B. GLCD $320 \times 240$}

There are 320 x 240 dots (pixels).Horizontal 320 pixels are divided into group of 8 bits and vertically 240 dots so as to contain total of 1200 characters on the screen. Real time parameters like input line to neutral voltage, input line to neutral current, input frequency, output line to line voltage, output line to neutral current, dc voltage etc. are displayed during Power ON screen. Home button is pressed to switch the screen to setting window. It is password protected. Setting of parameters is allowed only after entering the correct password. Once setting is completed, main screen displays modified values according to setting. Fig. 8. shows the programming of 320x240 GLCD.

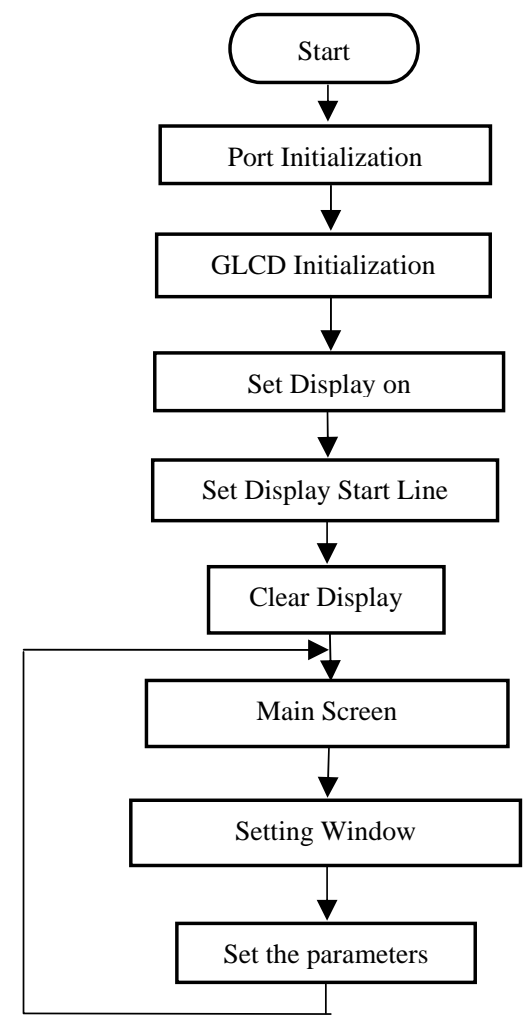

Fig. 8. Programming of 320x240 GLCD

\section{SNMP}

When first time SNMP card is connected to UPS system, network conditions are unknown, cannot configure the SNMP card. So USB configuration is used to read IP address of SNMP card. NMLUsb application file is executed to display IP address of SNMP card as shown in Fig. 9.

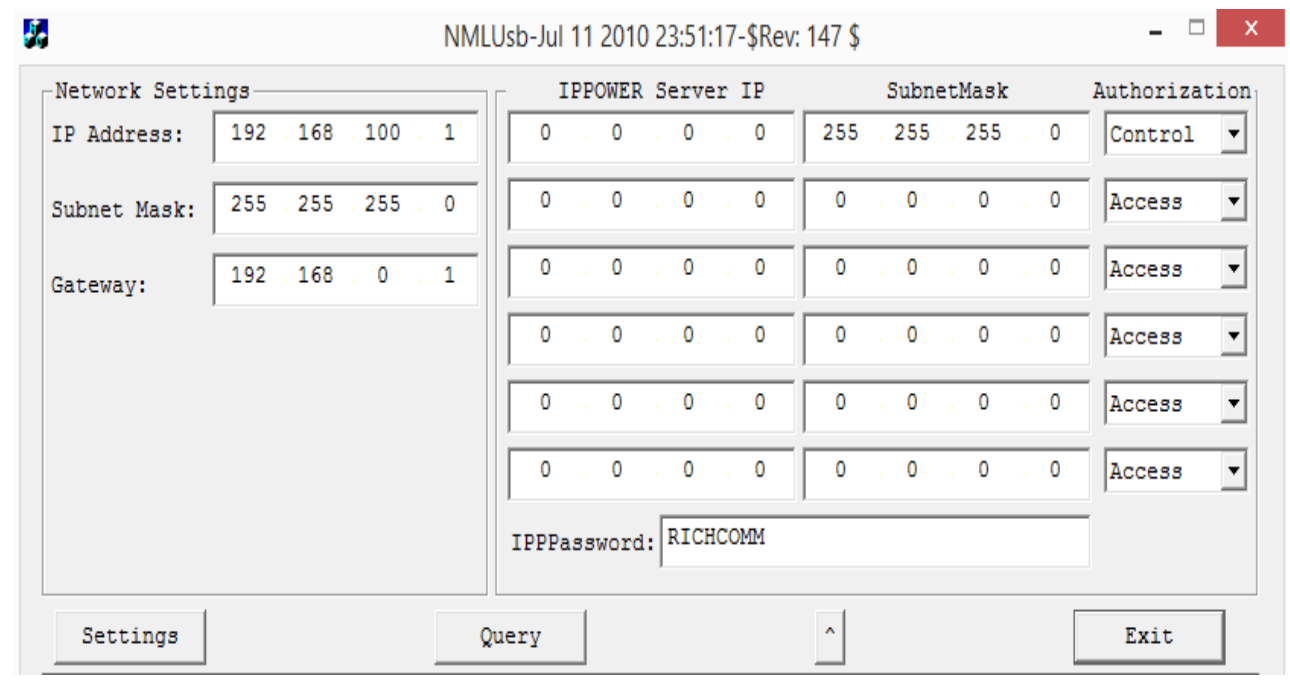

Fig. 9. USB Configuration 
SNMP card also supports the HTTP network protocol to access UPS system by Web browser. Set the IP address of Ethernet connected to SNMP card and manager PC as shown in Fig. 10.

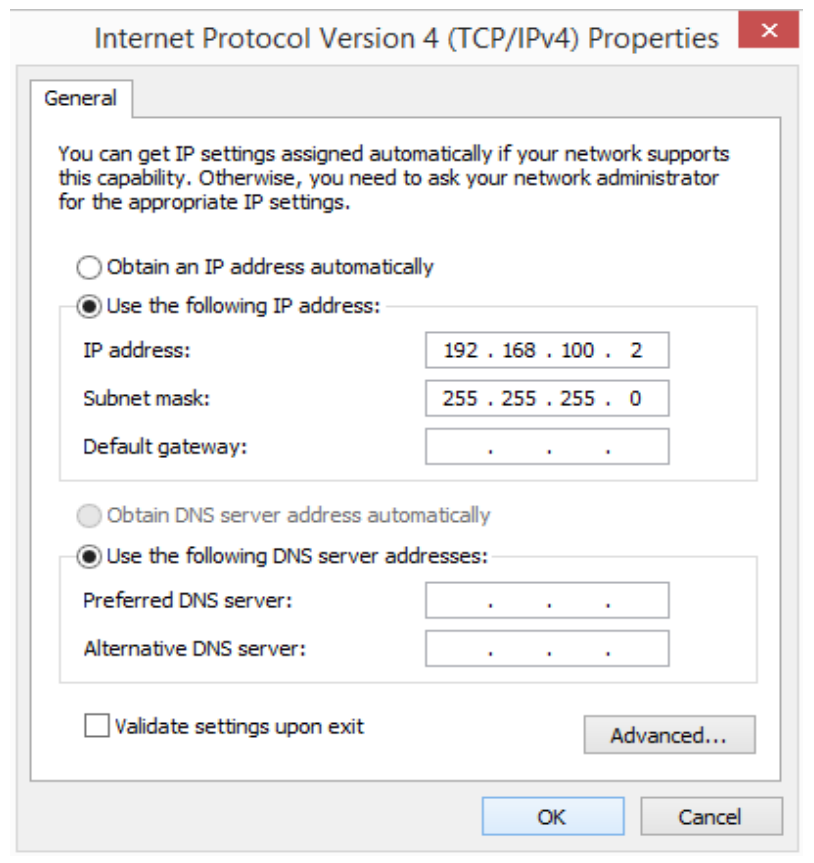

Fig. 10. Ethernet Configuration

V. Results

There are two modes namely mains operation mode and inverter operation mode. When input voltage ranges from $165 \mathrm{~V}$ to $255 \mathrm{~V}$, mains mode parameters are displayed. When input voltage goes below $105 \mathrm{~V}$ or exceeds $265 \mathrm{~V}$, inverter mode parameters are displayed. Fig. 11 \& 12 shows real time electrical parameters of Inverter when it is operated on mains and on battery supply respectively.

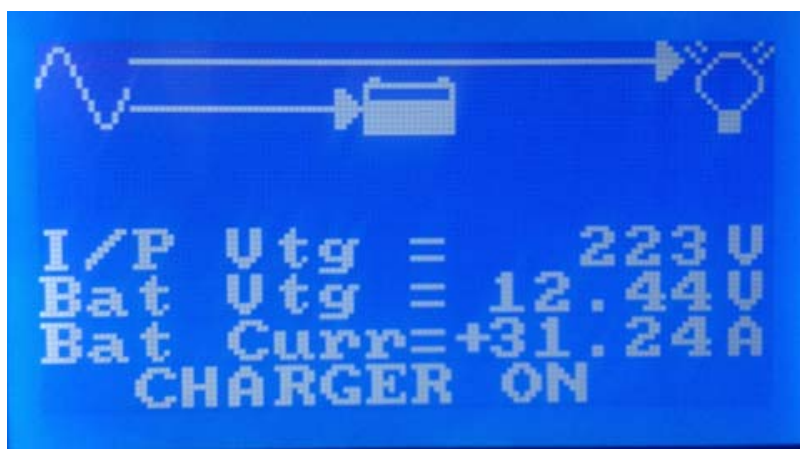

Fig. 11.Electrical parameters: Mains mode

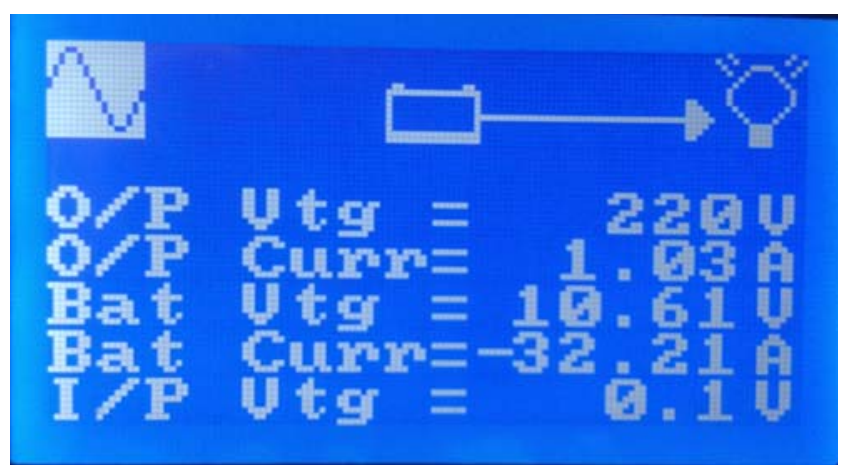

Fig. 12. Electrical parameters: Inverter mode 
In Inverter mode, if output Line and Neutral terminals get shorted, short-circuit warning should be displayed. If Battery voltage goes below $10.5 \mathrm{~V}$, battery low warning should be displayed. In Inverter mode, if load exceeds $110 \%(100 \%$ is equivalent to $600 \mathrm{~W})$, overload warning is displayed. If heat sink temperature exceeds $75^{\circ} \mathrm{C}$, over temperature warning is displayed. Fig. 13. shows various fault messages.

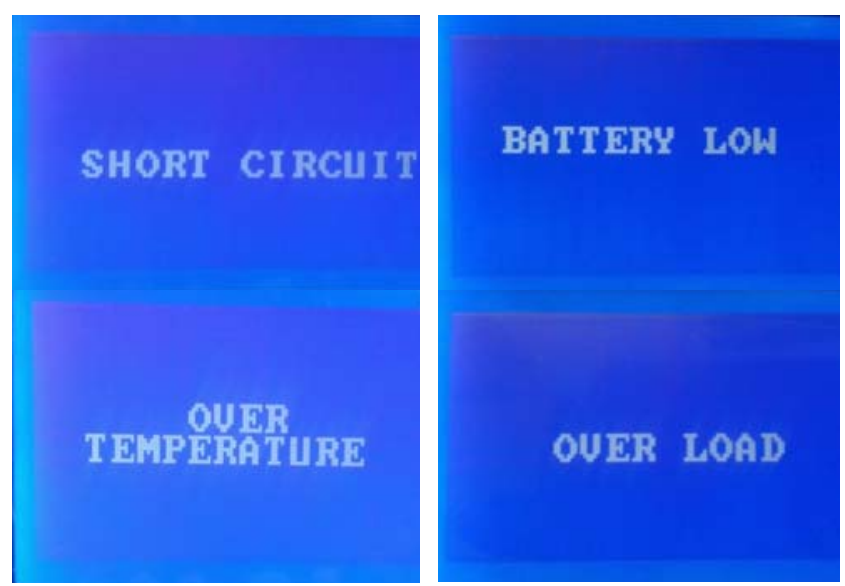

Fig. 13. Warning Messages

Main screen displays the real-time parameters of Static Voltage Regulator and Active Filter like input voltage, input current, input frequency, output voltage, output current, DC voltage and warning messages as shown in Fig. 14.

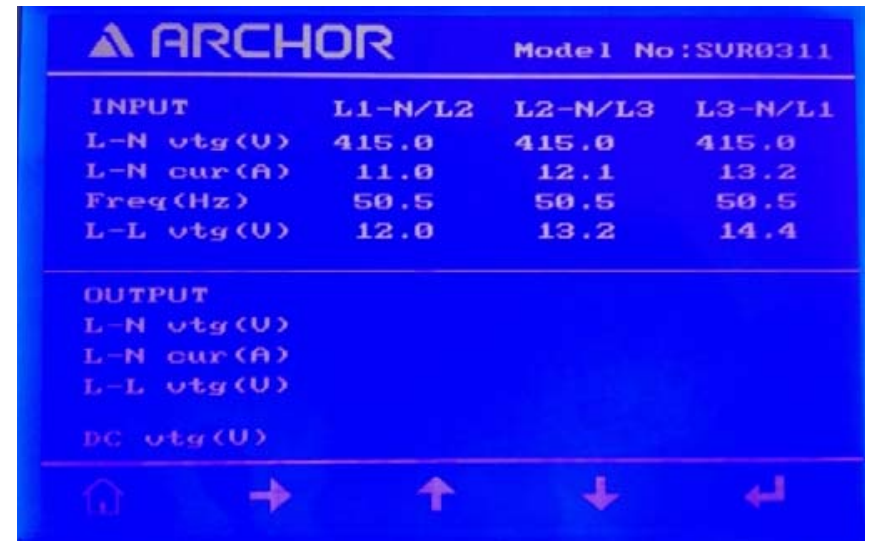

Fig. 14. Main Screen

Setting screen is used to set the system parameters like system voltage, input voltage range, output voltage range and output voltage. It is password protected. System parameters can be set only if correct password is entered as shown in Fig. 15.

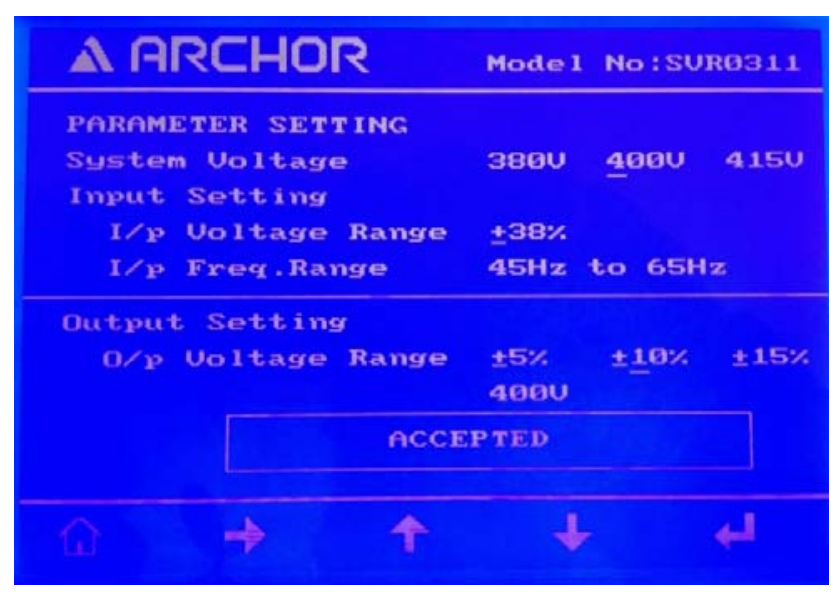

Fig. 15. Setting Screen 
Analog signal is converted into digital using A/D converter. Full analog waveform is converted into 400 digital samples ranging the ADC count from 0 to 1023.If the ADC count corresponds to the current sample is greater than the ADC count corresponds to previous sample, then waveform results into rising waveform. If ADC count corresponds to current sample is lower than ADC count corresponds to previous sample, waveform results into falling waveform as shown in Fig. 16.

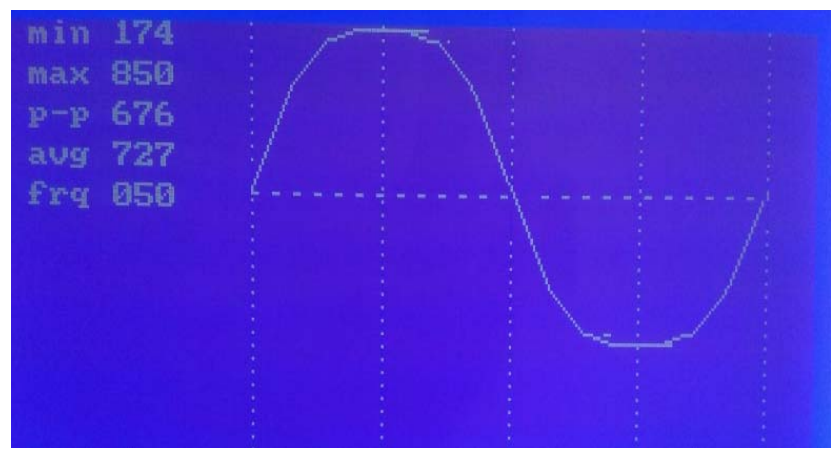

Fig. 16. Sinusoidal Waveform

Real-time electrical parameters of single phase inverter operated in mains mode \& inverter mode, are downloaded from microcontroller to the PC as shown in Fig. 17 \& Fig. 18.

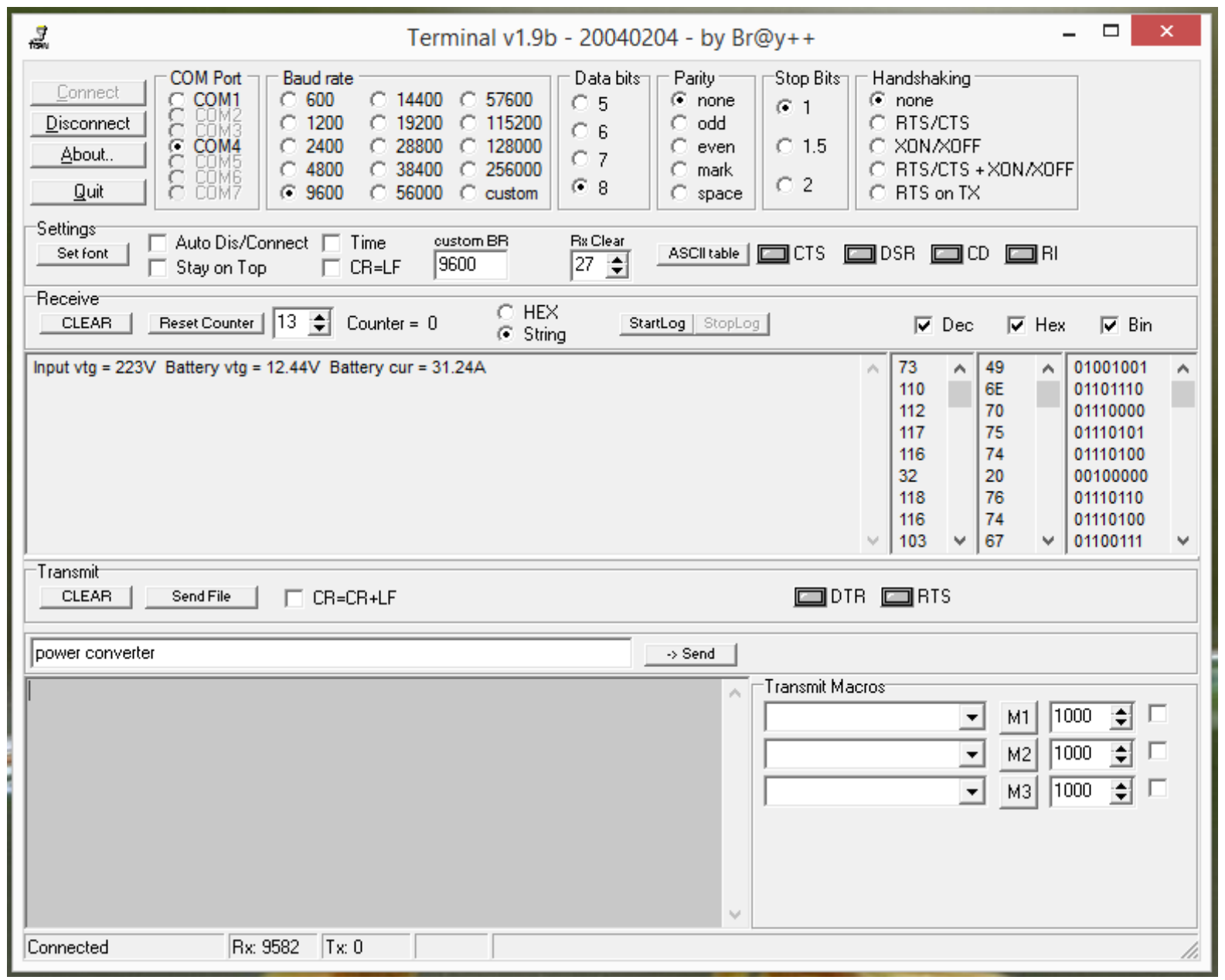

Fig. 17. Result of USB for Mains mode 


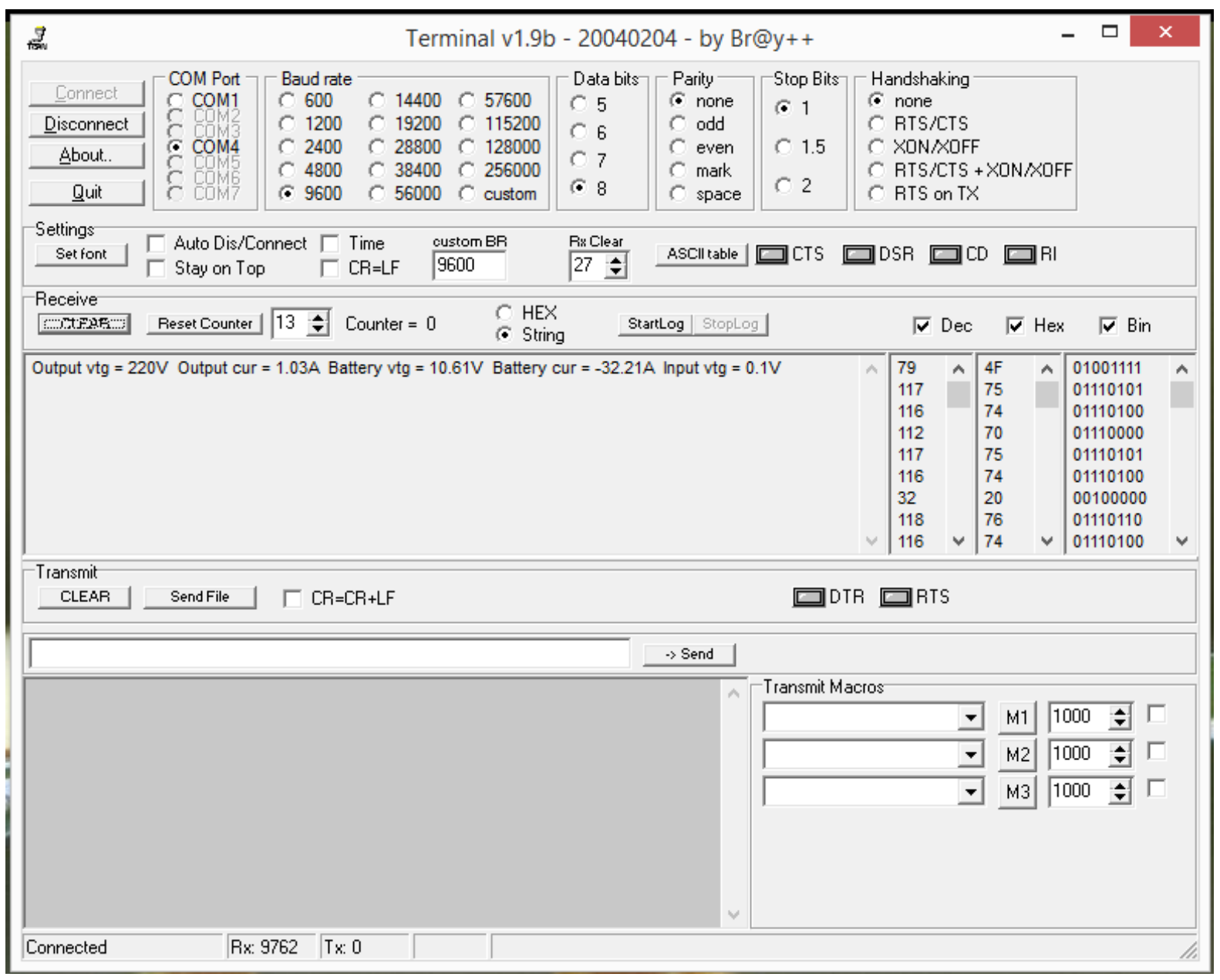

Fig. 18. Result of USB for Inverter mode

Real-time electrical parameters of UPS are monitored on Manager PC through GUI as shown in Fig. 19.

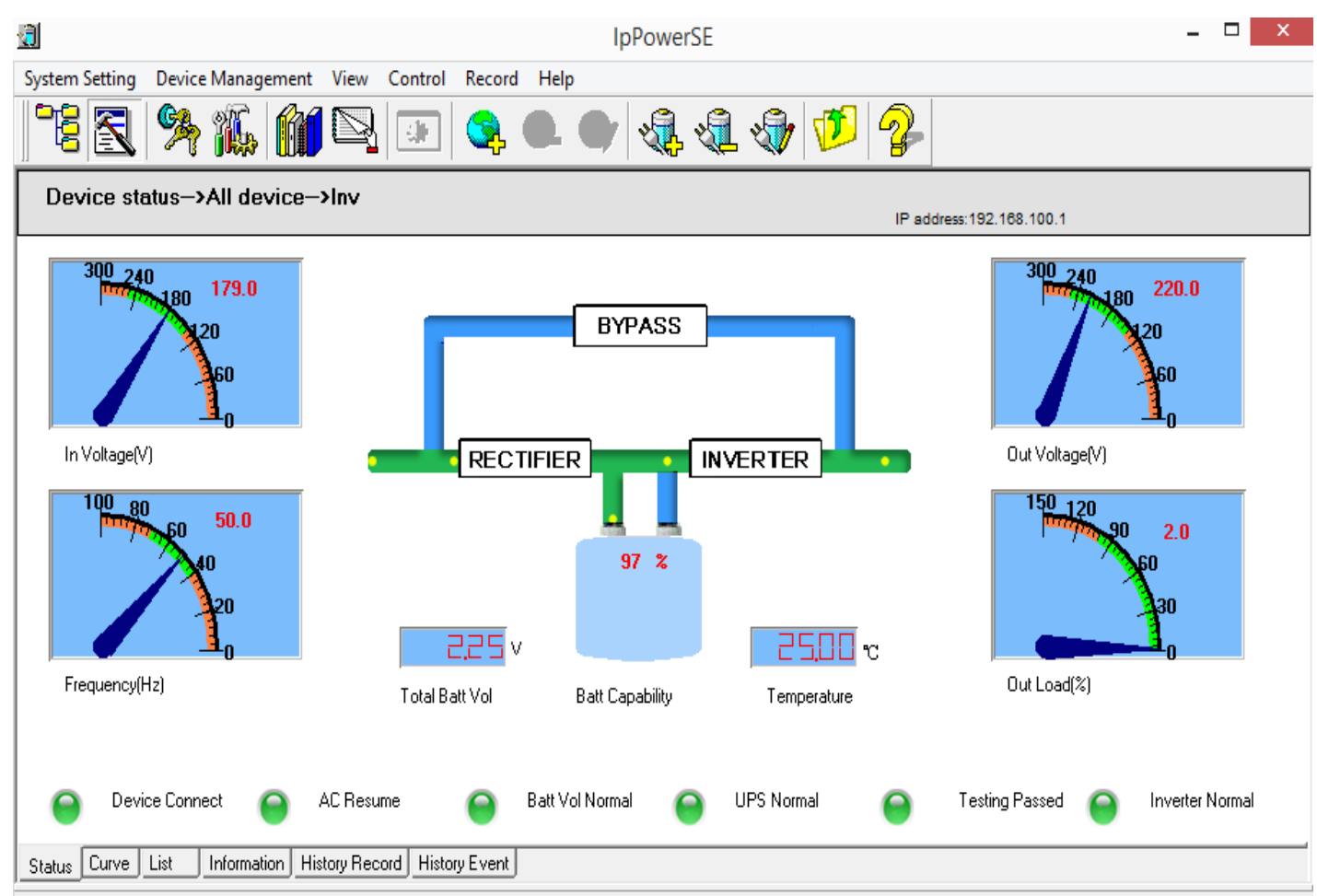

Fig. 19. Results on SNMP Manager 
UPS parameters can be viewed through web access by typing the IP address of SNMP card in the address bar of the web page as shown in Fig. 20.

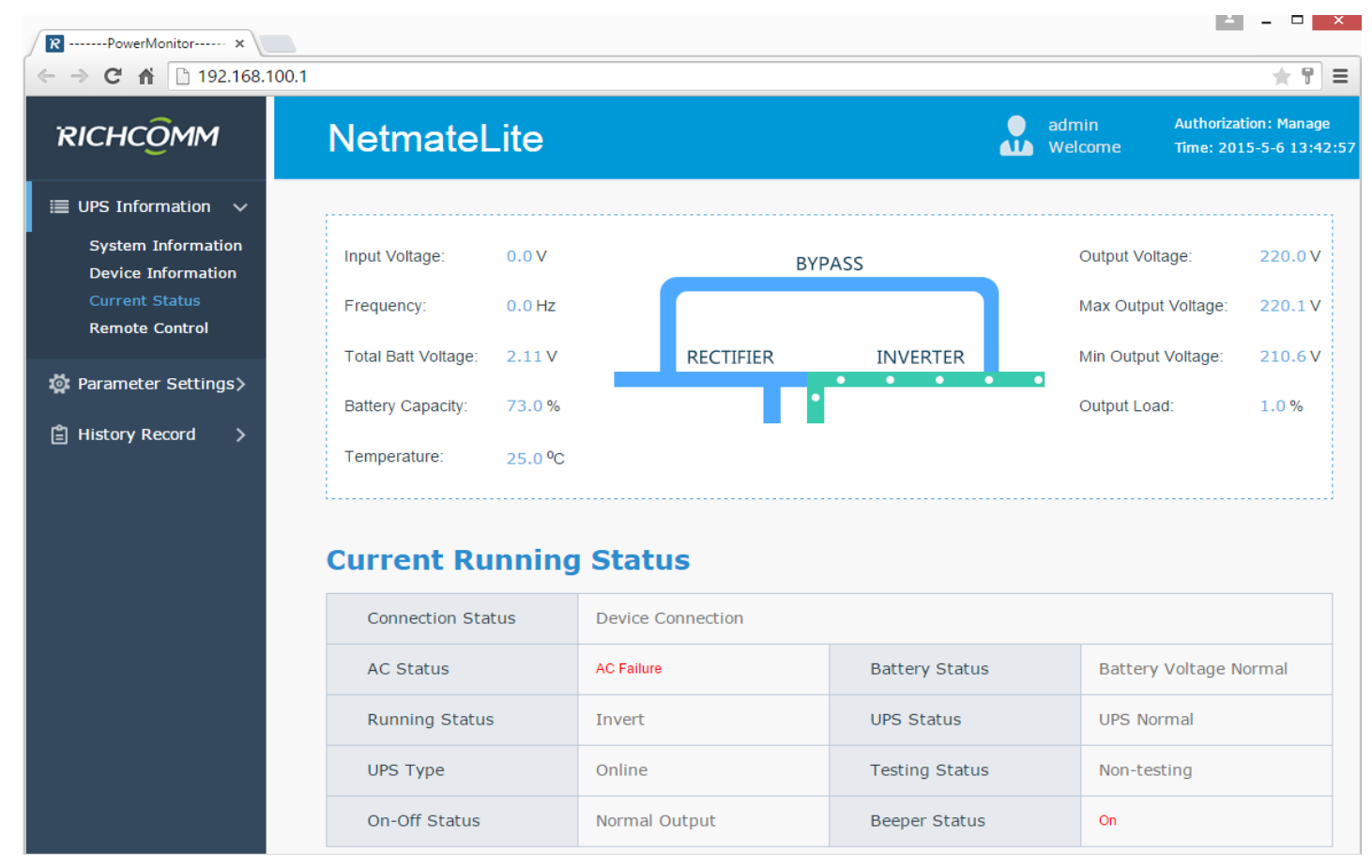

Fig. 20. Result on web

\section{CONCLUSION}

The communication interfaces GLCD, GSM, USB \& SNMP card with RL78/G14 microcontroller has been implemented to display and monitor real time parameters of power converters like Single Phase Inverter, UPS, Static Voltage Regulator and Active Filter. As per the requirements GLCD is interfaced to display electrical parameters and warnings. Sending of fault and warning messages through GSM is achieved. The electrical parameters of Single Phase Inverter are also transmitted to the PC using UART-to-USB converter. SNMP card is configured to UPS system and network connection. Single phase inverters are used for domestic as well as commercial purposes whereas Three Phase Active Filter \& Static Voltage Regulator are used in industry.

The future scope of this project could be wireless monitoring system using SNMP card for UPS. Designer can go for extranet protocol to achieve long-distance communication. However, the use of extranet protocol completely depends on the requirement of consumer. In case of current design, if user wants to access the database of parameters for power converter then user needs to connect an external computer with software support to read the data through USB. This seems to be a limitation, as user won't always possess a system to read the data. The future scope can include a hardware and software support to directly send the data to Storage Devices through USB as and when the user wants to read the database of parameters.

\section{REFERENCES}

[1] Neha R. Laddha, A.P.Thakare, "Implementation of serial communication using UART with configurable baud rate", International Journal on Recent and Innovation Trends in Computing and Communication, vol. 1, issue 4, pp. 263-268, 2013.

[2] Manisha Sharma, Nidhi Agarwal, S R N Reddy, "Design \& development of daughter board for USB-UART communication between Rasberry Pi and PC”, International Conference on Computing, Communication \& Automation, pp. 944-948, 2015.

[3] Saad Islam, Fatima Ajmal, Salman Ali, Jawad Zahid and Adnan Rashdi,"Secure End-to-End Communication over GSM and PSTN Networks",pp. 323-326, 2009.

[4] Xu Kun, Xiao Gang, Feng HAN, Dai Hao, ” Study of SNMP Network Management Interface Emulation Method”, 2009 Second International Conference on Information and Computing Science, pp. 207-210, 2009.

[5] Achmad Affandi, Dhany Riyanto, Istas Pratomo, Gatot Kusrahardjo, "Design \& Implementation fast response system monitoring system using Simple Network Management Protocol (SNMP)”, 2015 International Seminar on Intelligent Technology and its applications, pp. 385-390, 2015.

[6] Badotra, Sumit Singh, Japinder, “A review paper on Software Defined Networking”, International Journal of Advanced Research in Computer Science; Vol. 8, Issue. 3, 2017.

[7] Cube suite+ start-up guide Application note R01AN1232EJ0100 Rev.1.00 Aug. 24, 2012.

[8] RL78/G14 User’s Manual: Hardware 16-Bit Single-Chip Microcontrollers, Rev.1.00 Dec 2011, Renesas Electronics, www.renesas.com

[9] Universal Serial Bus Specification, Revision 2.0, Compaq, 2000 


\section{AUTHOR PROFILE}

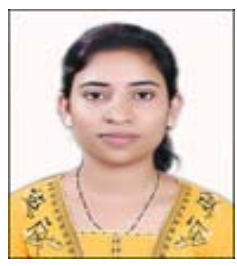

Snehal R. Watharkar completed her Bachelor of Technology in Electronics Engineering from Walchand College of Engineering, Sangli in 2012 and completed Masters of Technology in VLSI \& Embedded Systems from College of Engineering, Pune in 2015. She has worked as Engineer in Crompton Greaves, Mumbai and currently working as Assistant Professor in Rajarambapu Institute of Technology, Islampur, Sangli, Maharashtra.

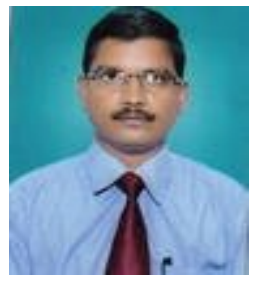

Mahadev S. Patil has received B.E.degree in Electronics and Communication Engineering from Karnataka University Dharwar in 1995, M. Tech. degree in Power Electronics from Indian Institute of Technology, Bombay Powai, Mumbai in 2001 and Ph.D. degree in Electronics and Telecommunication Engineering from Shivaji University, Kolhapur in 2014. He has 22 years of teaching experience. He has published more than 68 research papers and filed 02 patents. Currently he is working as Professor and Head of Electronics and Telecommunication Engineering in Rajarambapu Institute of Technology, Islampur, Sangli, Maharashtra. His field of interest includes power electronics, industrial control and instrumentation and telecommunication engineering. He is a member of Institution of Engineers (India), member of IETE New Delhi, Member of IET and life member of ISTE New Delhi. 